\title{
An approach to NLO QCD analysis of the semi-inclusive DIS data with modified Jacobi polynomial expansion method
}

\author{
A.N. Sissakian ${ }^{1}$, O.Yu. Shevchenko ${ }^{2}$, O.N. Ivanov ${ }^{3}$
}

Joint Institute for Nuclear Research

\begin{abstract}
It is proposed the modification of the Jacobi polynomial expansion method (MJEM) which is based on the application of the truncated moments instead of the full ones. This allows to reconstruct with a high precision the local quark helicity distributions even for the narrow accessible for measurement Bjorken $x$ region using as an input only four first moments extracted from the data in NLO QCD. It is also proposed the variational (extrapolation) procedure allowing to reconstruct the distributions outside the accessible Bjorken $x$ region using the distributions obtained with MJEM in the accessible region. The numerical calculations encourage one that the proposed variational (extrapolation) procedure could be applied to estimate the full first (especially important) quark moments.
\end{abstract}

The extraction of the quark helicity distributions is one of the main tasks of the semi-inclusive deep inelastic scattering (SIDIS) experiments (HERMES [1], COMPASS 2]) with the polarized beam and target. At the same time it was argued [3] that to obtain the reliable distributions at relatively low average $Q^{2}$ available to the modern SIDIS experiments ${ }^{4}$, the leading order (LO) analysis is not sufficient and next to leading order analysis (NLO) is necessary. In ref. [4 the procedure allowing the direct extraction from the SIDIS data of the first moments of the quark helicity distributions in NLO QCD was proposed. However, in spite of the special importance of the first moments, it is certainly very desirable to have the procedure of reconstruction in NLO QCD of the polarized densities themselves. However, it is extremely difficult to extract the local in $x_{B}$ distributions directly, because of the double convolution product entering the NLO QCD expressions for semi-inclusive asymmetries (see 4] and references therein). On the other hand, operating just as in ref. [4, one can directly extract not only the first moments, but the Mellin moments of any required order. The simple extension of the procedure proposed in ref. [4] gives for the $\mathrm{n}$-th moments $\Delta_{n} q \equiv \int_{0}^{1} d x x^{n-1} q(x)$ of the valence distributions the equations

$$
\Delta_{n} u_{V}=\frac{1}{5} \frac{\mathcal{A}_{p}^{(n)}+\mathcal{A}_{d}^{(n)}}{L_{(n) 1}-L_{(n) 2}} ; \quad \Delta_{n} d_{V}=\frac{1}{5} \frac{4 \mathcal{A}_{d}^{(n)}-\mathcal{A}_{p}^{(n)}}{L_{(n) 1}-L_{(n) 2}}
$$

where all quantities in the right-hand side are the same as in ref. [4] (see Eqs. (18-23)) with the replacement of $\int_{0}^{1} d x$ by $\int_{0}^{1} d x x^{n-1}$.

\footnotetext{
${ }^{1}$ E-mail address: sisakian@jinr.ru

${ }^{2}$ E-mail address: shevch@nusun.jinr.ru

${ }^{3}$ E-mail address: ivon@jinr.ru

${ }^{4}$ For example, HERMES data [1] on semi-inclusive asymmetries is obtained at $Q_{\text {average }}^{2}=2.5 \mathrm{GeV}^{2}$.
} 
It should be noticed that in reality one can measure the asymmetries only in the restricted $x_{B}$ region $a<x<b$, so that the approximate equations for the truncated moments

$$
\Delta_{n}^{\prime} q \equiv \int_{a}^{b} d x x^{n-1} q(x)
$$

of the valence distributions have a form (11) with the replacement of the full integrals by the sums over bins covering accessible $x_{B}$ region $a<x<b$, so that

$$
\left.\mathcal{A}_{p}^{(n)} \simeq \sum_{i=1}^{N_{\text {bins }}} x^{n-1} \Delta x_{i} A_{p}^{\pi^{+}-\pi^{-}}\left(x_{i}\right)\right|_{Z}\left(4 u_{V}-d_{V}\right)\left(x_{i}\right) \int_{Z}^{1} d z_{h}\left[1+\otimes \frac{\alpha_{s}}{2 \pi} \tilde{C}_{q q} \otimes\right]\left(D_{1}-D_{2}\right)
$$

and analogously for $\mathcal{A}_{d}^{(n)}$.

Thus, one can directly extract from the data the n-th Mellin moments of valence distributions. The question arises: is it sufficient to reconstruct the local in $x_{B}$ distributions?

There exist several methods allowing to reconstruct the local in $x_{B}$ quantities (like structure functions, polarized and unpolarized quark distributions, etc) knowing their n-th Mellin moments. All of them use the expansion of the local quantity in the series over the orthogonal polynomials (Bernstein, Legendre, Jacobi, etc). The most successful in applications (reconstruction of the local distributions from the evolved with GLAP moments and investigation of $\Lambda_{\mathrm{QCD}}$ ) occurred the Jacobi polynomial expansion method (JEM) proposed in the pioneer work by Parisi and Sourlas [5] and elaborated ${ }^{5}$ in refs. [6] and [7]. Within JEM the local in $x_{B}$ functions (structure functions or quark distributions) are expanded in the double series over the Jacobi polynomials and Mellin moments:

$$
F(x) \simeq F_{N_{\max }}(x)=x^{\beta}(1-x)^{\alpha} \sum_{k=0}^{N_{\max }} \Theta_{k}^{(\alpha, \beta)}(x) \sum_{j=0}^{k} c_{j}^{(k),(\alpha, \beta)} M[j+1]
$$

where $N_{\max }$ is the number of moments left ${ }^{6}$ in the expansion. For what follows it is of importance that the moments entering Eq. (44) are the full moments, i.e., the integrals over the entire $x_{B}$ region $0<x<1 M[j]=\int_{0}^{1} d x x^{j-1} F(x)$. Until now nobody investigated the question of applicability of JEM to the rather narrow $x_{B}$ region available to the modern polarized SIDIS experiments. So, let us try to apply JEM to the reconstruction of $\Delta u_{V}(x)$ and $\Delta d_{V}(x)$ in the rather narrow $x_{B}$ region $^{7} a=0.023<x<b=0.6$ available to HERMES, and to investigate is it possible to safely replace the full moments by the truncated ones. To this end we perform the simple test. We choose $^{8}$ GRSV2000NLO (symmetric sea) parametrization [9] at $Q^{2}=2.5 \mathrm{GeV}^{2}$. Integrating the parametrization over the HERMES $x_{B}$ region we then calculate twelve truncated moments of the $u$ and $d$ valence distributions given by Eq. (2) with $a=0.023, b=0.6$. Substituting these moments in expansion (4) with $N_{\max }=12$, we look for optimal values of parameters $\alpha$ and $\beta$ corresponding to the minimal deviation of

\footnotetext{
${ }^{5} \mathrm{JEM}$ with respect to polarized quark densities was first applied in ref. 8 .

${ }^{6}$ Expansion (4) becomes exact when $N_{\max } \rightarrow \infty$. However, the advantage of JEM is that even truncated series with small number of used moments $N_{\max }$ and properly fixed parameters $\alpha, \beta$ gives the good results (see, for example, [7]).

${ }^{7}$ We choose here the most narrow HERMES $x_{B}$ region where the difference between JEM and its modification MJEM (see below) application becomes especially impressive. However, even with the more wide $x_{B}$ region (for example, COMPASS 2] region $0.003<x<0.7$ ) it is of importance to avoid the additional systematical errors caused by the replacement of the full (unaccessible) moments in JEM (4) by the accessible truncated moments.

${ }^{8}$ Certainly, one can choose for testing any other parametrization.
} 
reconstructed curves for $\Delta u_{V}(x)$ and $\Delta d_{V}(x)$ from the input (reference) curves corresponding to input parametrization. To find these optimal values $\alpha_{\text {opt }}$ and $\beta_{\text {opt }}$ we use the program MINUIT [10. The results are presented in Fig. 1, where one can see that the curves strongly differ from each other even for the high number of used moments $N_{\max }=12$. Thus, the substitution of truncated moments instead of exact ones in the expansion (4) is a rather crude approximation at least for HERMES $x_{B}$ region. Fortunately it is possible to modify the standard JEM in a such way that new series contains the truncated moments instead of the full ones. The new expansion looks as (see the Appendix)

$$
\begin{aligned}
& F(x) \simeq F_{N_{\max }}(x)=\left(\frac{x-a}{b-a}\right)^{\beta}\left(1-\frac{x-a}{b-a}\right)^{\alpha} \\
& \times \sum_{n=0}^{N_{\max }} \Theta_{n}^{(\alpha, \beta)}\left(\frac{x-a}{b-a}\right) \sum_{k=0}^{n} c_{n k}^{(\alpha, \beta)} \frac{1}{(b-a)^{k+1}} \sum_{l=0}^{k} \frac{k !}{l !(k-l) !} M^{\prime}[l+1](-a)^{k-l},
\end{aligned}
$$

where we introduce the notation (c.f. Eq. (2))

$$
M^{\prime}[j] \equiv M_{[a, b]}^{\prime}[j] \equiv \int_{a}^{b} d x x^{j-1} F(x)
$$

for the moments truncated to accessible for measurement $x_{B}$ region. It is of great importance that now in the expansion enter not the full (unavailable) but the truncated (accessible) moments. Thus, having at our disposal few first truncated moments extracted in NLO QCD (see Eqs. (10)), and using MJEM (Eq. (15)), one can reconstruct the local distributions in the accessible for measurement $x_{B}$ region.

To proceed let us clarify the important question about the boundary distortions. The deviations of reconstructed with MJEM, Eq. (15), $F_{N_{\max }}$ from F near the boundary points are unavoidable since MJEM is correctly defined in the entire region $(a, b)$ except for the small vicinities of boundary points (see the Appendix). Fortunately, $F_{N_{\max }}$ and $\mathrm{F}$ are in very good agreement in the practically entire accessible $x_{B}$ region, while the boundary distortions are easy identified and controlled since they are very sharp and hold in very small vicinities of the boundary points (see Figs. 2-5 below). Performing the extrapolation outside the accessible $x_{B}$ region one just should cut off these unphysical boundary distortions (see below).

Let us check how well MJEM works. To this end let us repeat the simple exercises with reconstruction of the known GRSV2000NLO (symmetric sea) parametrization and compare the results of $\Delta u_{V}(x)$ and $\Delta d_{V}(x)$ reconstruction with the usual JEM and the proposed MJEM. To control the quality of reconstruction we introduce the parameter ${ }^{9}$

$$
\nu=\frac{\int_{a}^{b} d x\left|F_{\text {reconstructed }}(x)-F_{\text {reference }}(x)\right|}{\left|\int_{a}^{b} d x F_{\text {reference }}\right|} \cdot 100 \%,
$$

where $F_{\text {reference }}(x)$ corresponds to the input parametrization and $F_{\text {reconstructed }}(x) \equiv F_{N_{\max }}(x)$ in Eq. (5). We first perform the reconstruction with very high number of moments $N_{\max }=12$ (the maximum number of moments used with standard JEM in literature - see [7] and references therein) and then with small number $N_{\max }=4$. Notice that the last choice $N_{\max }=4$ is

\footnotetext{
${ }^{9}$ Calculating $\nu$ we just cut off the boundary distortions which hold for MJEM in the small vicinities of the boundary points (see the Appendix), and decrease the integration region, respectively. To be more precise, one can apply after cutting some extrapolation to the boundary points. However, the practice shows that the results on $\nu$ calculation are practically insensitive to the way of extrapolation since the widthes of the boundary distortion regions are very small (about $10^{-3}$ ).
} 
especially important because of peculiarities of the data on asymmetries provided by the SIDIS experiments. Indeed, the number of used moments should be as small as possible because first, the relative error $\left|\delta\left(M^{\prime}[j]\right) / M^{\prime}[j]\right|$ on $M^{\prime}[j]$ becomes higher with increase of $j$ and second, the high moments becomes very sensitive to the replacement of integration by the sum over the bins. The results of $\Delta u_{V}(x)$ and $\Delta d_{V}(x)$ reconstruction with MJEM at $N_{\max }=12$ and with both JEM and MJEM (in comparison) at $N_{\max }=4$, are presented by Figs. 2 and 3. It is seen (see Fig. 2) that for $N_{\max }=12$ MJEM, on the contrary to the usual JEM (see Fig. 1), gives the excellent agreement between the reference and reconstructed curves. In the case $N_{\max }=4$ the difference in quality of reconstruction between JEM and MJEM (see Fig. 3) becomes especially impressive. While for standard JEM the reconstructed and reference curves strongly differ from each other, the respective curves for MJEM are in a good agreement. Thus, one can conclude that dealing with the truncated, available to measurement, $x_{B}$ region one should apply the proposed modified JEM to obtain the reliable results on the local distributions.

Until now we looked for the optimal values of parameters $\alpha$ and $\beta$ entering MJEM using explicit form of the reference curve (input parametrization). Certainly, in reality we have no any reference curve to be used for optimization. However, one can extract from the data in NLO QCD the first few moments (see Eqs. (11)). Thus, we need some criterium of MJEM optimization which would use for optimization of $\alpha$ and $\beta$ only the known (extracted) moments entering MJEM.

On the first sight it seems to be natural to find the optimal values of $\alpha$ and $\beta$ minimizing the difference of reconstructed with MJEM and input ${ }^{10}$ (entering MJEM expansion (5) ) moments. However, it is easy to prove ${ }^{11}$ that this difference is equal to zero identically:

$$
\left.\left.M_{[a, b]}^{\prime}[n]\right|_{\text {reconstructed }} \equiv M_{[a, b]}^{\prime}[n]\right|_{\text {input }}, \quad n \leq N_{\max }
$$

i.e. all reconstructed moments with $n \leq N_{\max }$ are identically equal to the respective input moments for any $\alpha$ and $\beta$. Fortunately, we can use for comparison the reference "twicetruncated" moments

$$
M^{\prime \prime}[n] \equiv M_{\left[a+a^{\prime}, b-b^{\prime}\right]}^{\prime \prime}[n] \equiv \int_{a+a^{\prime}}^{b-b^{\prime}} d x x^{n-1} F(x) \quad\left(a<a+a^{\prime}<b-b^{\prime}<b\right),
$$

i.e. the integrals over the region less than the integration region $[a, b]$ for the "once-truncated" moments $M_{[a, b]}^{\prime}$ entering MJEM (5). The respective optimization criterium can be written in the form

$$
\sum_{j=0}^{N_{\max }}\left|M_{(\text {reconstructed })}^{\prime \prime}[j]-M_{(\text {reference) }}^{\prime \prime}[j]\right|=\min .
$$

The "twice truncated" reference moments should be extracted in NLO QCD from the data in the same way as the input (entering MJEM (5) ) "once truncated" moments. In reality one can obtain "twice-truncated" moments using Eqs. (II) and removing, for example, first and/or last bin from the sum in Eq. (3).

\footnotetext{
${ }^{10}$ In practice one should reconstruct these input moments from the data using Eqs. (1D). The reference "twice-truncated" moments (9) should be reconstructed from the data in the same way.

${ }^{11}$ It can be proved by analogy with the case of the usual JEM, where Eq. (8) with $[a, b]=[0,1]$ holds (see, for example, [7]).
} 
Let us now check how well the optimization criterium (10) works. To this end we again perform the simple numerical test. We choose GRSV2000NLO parametrization at $Q^{2}=2.5 \mathrm{GeV}^{2}$ with both broken and symmetric sea scenarios. We then calculate four first "once-truncated" and four first "twice-truncated" moments defined by Eqs. (6) and (9), and substitute them in the optimization criterium (10). To find the optimal values of $\alpha$ and $\beta$ we use the MINUIT 10 program. The results are presented by Fig. 4. It is seen that the optimization criterium works well for both symmetric and broken sea scenarios.

Thus, one can conclude that MJEM can be successfully applied for reconstruction of the local distributions knowing only few first truncated Mellin moments. Notice, however, that by construction MJEM reproduces the local distributions only in the accessible for measurement $x_{B}$ region. The question arises: could one attempt to reconstruct the local distributions outside the accessible region (i.e. to perform extrapolation) using the obtained with MJEM distributions as an input? To this end we propose to solve the following variational task. We apply MJEM, Eq. (5) , to the maximally ${ }^{12}$ extended $x_{B}$ region $\left[a_{\min }, b_{\max }\right]$ replacing the moments $M_{\left[a_{\min }, b_{\max }\right]}^{\prime}[j]$ by $M_{[a, b]}^{\prime}[j]+\epsilon_{j}$, where $\epsilon_{j}(j=1 \ldots 4)$ are the free variational parameters $\left(\epsilon_{j}\right.$ should be considered as unknown "tails" of the full moments). Then, using MINUIT program [10, one finds the parameters $\epsilon_{j}$ requiring the minimal deviation of the reconstructed with $\epsilon_{j}$ curve from the input (reconstructed with criterium (10) ) curve inside the accessible for measurement region $[a, b]$. The reconstructed in this way quantities $M_{[a, b]}^{\prime}[j]+\epsilon_{j}$ should be compared with the reference (obtained by direct integration of the input parametrization) moments $\left.M_{\left[a_{\min }, b_{\max }\right]}^{\prime}[j]\right|_{\text {reference }}$. In ideal case (ideal reconstruction of "tails" $\epsilon_{j}$ ) these quantities would coincide.

Let us test this variational (extrapolation) procedure by the simple numerical exercise. We choose GRSV2000 NLO parametrization (for both broken and symmetric sea scenarios) at $Q^{2}=2.5 \mathrm{GeV}^{2}$ as the reference one. Since the allowed [9] $x_{B}$ region for this parametrization is $\left[10^{-4}, 1\right]$ we choose $\left[a_{\min }, b_{\max }\right]=\left[10^{-4}, 1\right]$ and for the truncated region $[a, b]$ we again choose the accessible for HERMES $x_{B}$ region $[a, b]=[0.023,0.6]$. Notice that performing the variational (extrapolation) procedure we cut off the boundary distortions of the curve (which enters the variational procedure as an input) obtained with MJEM and criterium (10) inside the accessible $x_{B}$ region. The results of the variational (extrapolation) procedure application are presented by Fig 5 and Table 1 .

Comparing the reconstructed and input curves (see Fig. 5) one can see that they are in a quite good agreement while the slight deviation should be corrected in the future by improving the variational (extrapolation) procedure. Fortunately, all four moments occur almost insensitive to this deviation. Indeed, from the Table 1 it is seen that the reconstructed full moments for both $u$ and $d$ valence quarks and for both scenarios are in a good agreement with the respective reference values. The most interesting is that the reconstructed first moments which are of the most importance for understanding the proton spin puzzle are in good agreement with their reference values.

Thus, all numerical tests confirm that the proposed modification of the Jacobi polynomial expansion method, MJEM, allows to reconstruct with a high precision the quark helicity distributions in the accessible for measurement $x_{B}$ region. Besides, the numerical calculations demonstrate that the application of MJEM together with the special variational (extrapolation) procedure can allow to estimate the full first (especially important) quark moments knowing the distributions reconstructed with MJEM in the accessible for measurement $x_{B}$ region.

\footnotetext{
${ }^{12}$ For a moment, we restrict ourselves by the $x_{B}$ region $\left[a_{\min }=10^{-4}, b_{\max }=1\right]$ which is typical for the most known parametrizations on the quark helicity distributions.
} 
Table 1: Results of first four moments of $\Delta u_{V}$ and $\Delta d_{V}$ reconstruction in the region $\left[a_{\min }=\right.$ $\left.10^{-4}, b_{\max }=1\right]$ for the GRSV2000NLO parametrization for both symmetric (top) and broken sea (bottom) scenarios.

\begin{tabular}{cccc|ccc}
\hline \hline & & $\Delta u_{V}(x)$ & \multicolumn{3}{c}{$\Delta d_{V}(x)$} \\
$\mathrm{n}$ & $M_{[0.023,0.6]}^{\text {'input }}$ & $M_{\left[10^{-4}, 1\right]}^{\text {out }}$ & $M_{\left[10^{-4}, 1\right]}^{\prime \text { referen }}$ & $M_{[0.023,0.6]}^{\text {input }}$ & $M_{\left[10^{-4}, 1\right]}^{\text {out }}$ & $M_{\left[10^{-4}, 1\right]}^{\prime \text { referece }}$ \\
\hline 1 & 0.749 & 0.904 & 0.917 & -0.275 & -0.362 & -0.340 \\
2 & 0.153 & 0.164 & 0.167 & -0.049 & -0.051 & -0.051 \\
3 & 0.047 & 0.053 & 0.055 & -0.013 & -0.014 & -0.014 \\
4 & 0.017 & 0.021 & 0.023 & -0.004 & -0.005 & -0.005 \\
\hline \hline 1 & 0.570 & 0.609 & 0.605 & -0.114 & -0.074 & -0.029 \\
2 & 0.137 & 0.150 & 0.149 & -0.036 & -0.038 & -0.037 \\
3 & 0.044 & 0.052 & 0.052 & -0.012 & -0.013 & -0.013 \\
4 & 0.017 & 0.023 & 0.022 & -0.004 & -0.006 & -0.005 \\
\hline
\end{tabular}

The authors are grateful to R. Bertini, O. Denisov, A. Korzenev, V. Krivokhizhin, E. Kuraev, A. Maggiora, A. Nagaytsev, A. Olshevsky, G. Piragino, G. Pontecorvo, I. Savin, A. Sidorov and O. Teryaev, for fruitful discussions. Two of us (O.S, O.I.) thanks RFBR grant 05-02-17748.

\section{Appendix}

The JEM is the expansion of the $x$-dependent function (structure function or quark density) in the series over Jacobi polynomials $\Theta_{n}^{(\alpha, \beta)}(x)$ orthogonal with weight $\omega^{(\alpha, \beta)}(x)=x^{\beta}(1-x)^{\alpha}$ (see [5] - [7] for details):

$$
F(x)=\omega^{(\alpha, \beta)} \sum_{k=0}^{\infty} \Theta_{k}^{(\alpha, \beta)}(x) \sum_{j=0}^{k} c_{k j}^{(\alpha, \beta)} M(j+1),
$$

where

$$
M[j]=\int_{0}^{1} d x x^{j-1} F(x)
$$

and

$$
\int_{0}^{1} d x \omega^{(\alpha, \beta)}(x) \Theta_{n}^{(\alpha, \beta)}(x) \Theta_{m}^{(\alpha, \beta)}(x)=\delta_{n m} .
$$

The details on the Jacobi polynomials

$$
\Theta_{k}^{(\alpha, \beta)}(x)=\sum_{j=0}^{k} c_{k j}^{(\alpha, \beta)} x^{j}
$$

can be found in refs. [5] and [6]. In practice one truncates the series (A.4) living in the expansion only finite number of moments $N_{\max }$ - see Eq. (5). The experience shows [7] that JEM produces good results even with small number $N_{\max }$.

The idea of modified expansion is to reexpand $F(x)$ in the series over the truncated moments $M_{[a b]}^{\prime}[j]$ given by Eq. (6) $)$, performing the rescaling $x \rightarrow a+(b-a) x$ which compress the entire 
region $[0,1]$ to the truncated region $[a, b]$. To this end let us apply the following ansatz ${ }^{13}$

$$
F(x)=\left(\frac{x-a}{b-a}\right)^{\beta}\left(1-\frac{x-a}{b-a}\right)^{\alpha} \sum_{n=0}^{\infty} \tilde{f}_{n} \Theta_{n}^{(\alpha, \beta)}\left(\frac{x-a}{b-a}\right)
$$

and try to find the coefficients $\tilde{f}_{n}$. Multiplying both parts of Eq. (A.5) by $\Theta_{k}^{(\alpha, \beta)}((x-a) /(b-a))$, integrating over $\mathrm{x}$ in the limits $[a, b]$ and performing the replacement $t=(x-a) /(b-a)$, one gets

$$
\int_{a}^{b} d x F(x) \Theta_{k}^{(\alpha, \beta)}\left(\frac{x-a}{b-a}\right)=(b-a) \sum_{n=0}^{\infty} \tilde{f}_{n} \int_{0}^{1} d t t^{\beta}(1-t)^{\alpha} \Theta_{n}^{(\alpha, \beta)}(t) \Theta_{k}^{(\alpha, \beta)}(t),
$$

so that with the orthogonality condition Eq. (A.3) one obtains

$$
\tilde{f}_{n}=(b-a)^{-1} \int_{a}^{b} d x F(x) \Theta_{n}^{(\alpha, \beta)}\left(\frac{x-a}{b-a}\right) .
$$

Substituting Eq. (A.7) in the expansion (A.5), and using Eq. (A.4) one eventually gets

$$
\begin{aligned}
& F(x)=\left(\frac{x-a}{b-a}\right)^{\beta}\left(1-\frac{x-a}{b-a}\right)^{\alpha} \\
& \times \sum_{n=0}^{\infty} \Theta_{n}^{(\alpha, \beta)}\left(\frac{x-a}{b-a}\right) \sum_{k=0}^{n} c_{n k}^{(\alpha, \beta)} \frac{1}{(b-a)^{k+1}} \sum_{l=0}^{k} \frac{k !}{l !(k-l) !} M_{[a, b]}^{\prime}[l+1](-a)^{k-l},
\end{aligned}
$$

where $M_{[a, b]}^{\prime}[j]$ is given by Eq. (6). Truncating in the exact Eq. (A.8) the infinite sum over $n$ to the $\operatorname{sum} \sum_{n=0}^{N_{\max }}$ one gets the approximate equation (15).

\section{References}

[1] HERMES collaboration (A. Airapetyan et al.), Phys. Rev. D71 (2005) 012003

[2] COMPASS collaboration (G. Baum et al.), "COMPASS: A proposal for a common muon and proton apparatus for structure and spectroscopy", CERN-SPSLC-96-14 (1996).

[3] A.N. Sissakian, O.Yu. Shevchenko and O.N. Ivanov, Phys. Rev. D68 (2003) 031502

[4] A.N. Sissakian, O.Yu. Shevchenko and O.N. Ivanov, Phys. Rev. D70 (2004) 074032

[5] G. Parisi, N. Sourlas, Nucl. Phys. B151 (1979) 421

[6] I.S. Barker, C.S. Langensiepen, G. Shaw, Nucl. Phys. B186 (1981) 61; CERN-TH-2988

[7] V.G. Krivokhizhin et al, Z. Phys. C36 (1987) 51; JINR-E2-86-564

[8] E. Leader, A.V. Sidorov, D.B. Stamenov, Int. J. Mod. Phys. A13 (1998) 5573

[9] M. Gluck, E. Reya, M. Stratmann, W. Vogelsang, Phys. Rev. D63 (2001) 094005; hep-ph/0011215

[10] F. James, M. Roos, Comput. Phys. Commun. 10 (1975) 343

\footnotetext{
${ }^{13}$ Notice that ansatz (A.5) (as well as the expansion Eq. (5) itself) is correctly defined inside the entire region $(a, b)$ except for the small vicinities of boundary points (absolutely the same situation holds for the usual JEM, Eq. A.1 , applied to the quark distributions in the region $(0,1))$. In practice, the respective boundary distortions are just cut off when one performs the extrapolation procedure.
} 
Figure 1: The results of reconstruction of $\Delta u_{V}(x)\left(\alpha_{\text {opt }}=8.18922, \beta_{\text {opt }}=-0.99\right)$ and $\Delta d_{V}(x)$ $\left(\alpha_{\text {opt }}=-0.99, \beta_{\text {opt }}=-0.387196\right)$ with the usual JEM. Solid line corresponds to input (reference) parametrization. Dotted line corresponds to the distributions reconstructed with JEM
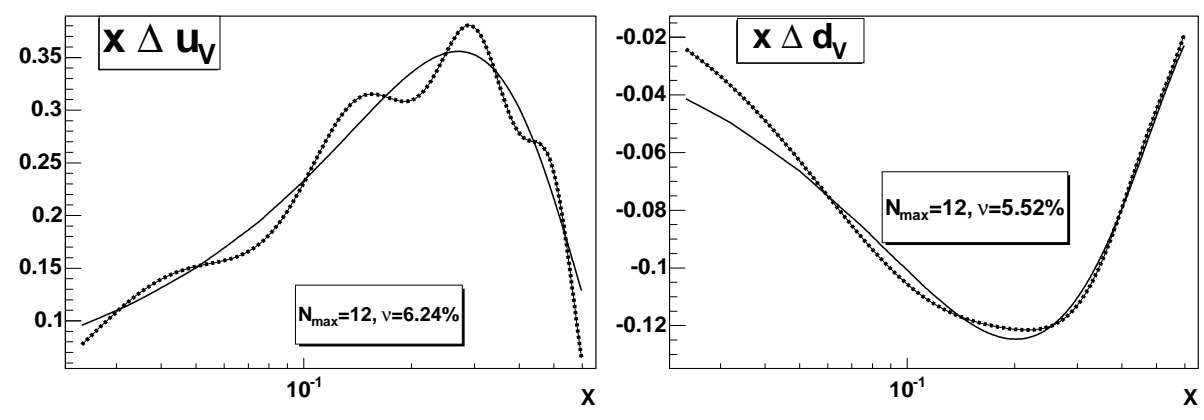

Figure 2: The results of $\Delta u_{V}(x)\left(\alpha_{o p t}=-0.827885, \beta_{\text {opt }}=-0.011505\right)$ and $\Delta d_{V}(x)\left(\alpha_{o p t}=\right.$ $\left.-0.989752, \beta_{\text {opt }}=-0.012393\right)$ reconstruction with MJEM. Solid line corresponds to input (reference) parametrization. Dotted line corresponds to the distributions reconstructed with MJEM .
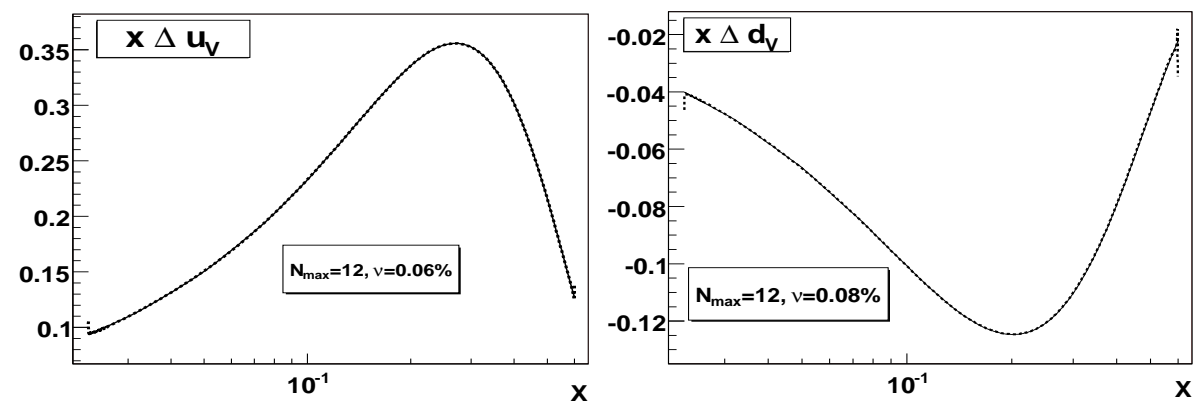
Figure 3: The top part corresponds to $\Delta u_{V}(x)\left(\alpha_{\text {opt }}=-0.99, \beta_{\text {opt }}=0.054010\right)$ and $\Delta d_{V}(x)$ $\left(\alpha_{\text {opt }}=0.174096, \beta_{\text {opt }}=0.162567\right)$ reconstructed with the usual JEM. The bottom part corresponds to $\Delta u_{V}(x)\left(\alpha_{o p t}=-0.0025869, \beta_{\text {opt }}=-0.071591\right)$ and $\Delta d_{V}(x)\left(\alpha_{o p t}=0.110331\right.$, $\left.\beta_{\text {opt }}=-0.049255\right)$ reconstructed with MJEM. Solid lines correspond to input (reference) parametrization. Dotted lines correspond to the distributions reconstructed with JEM (top) and MJEM (bottom).
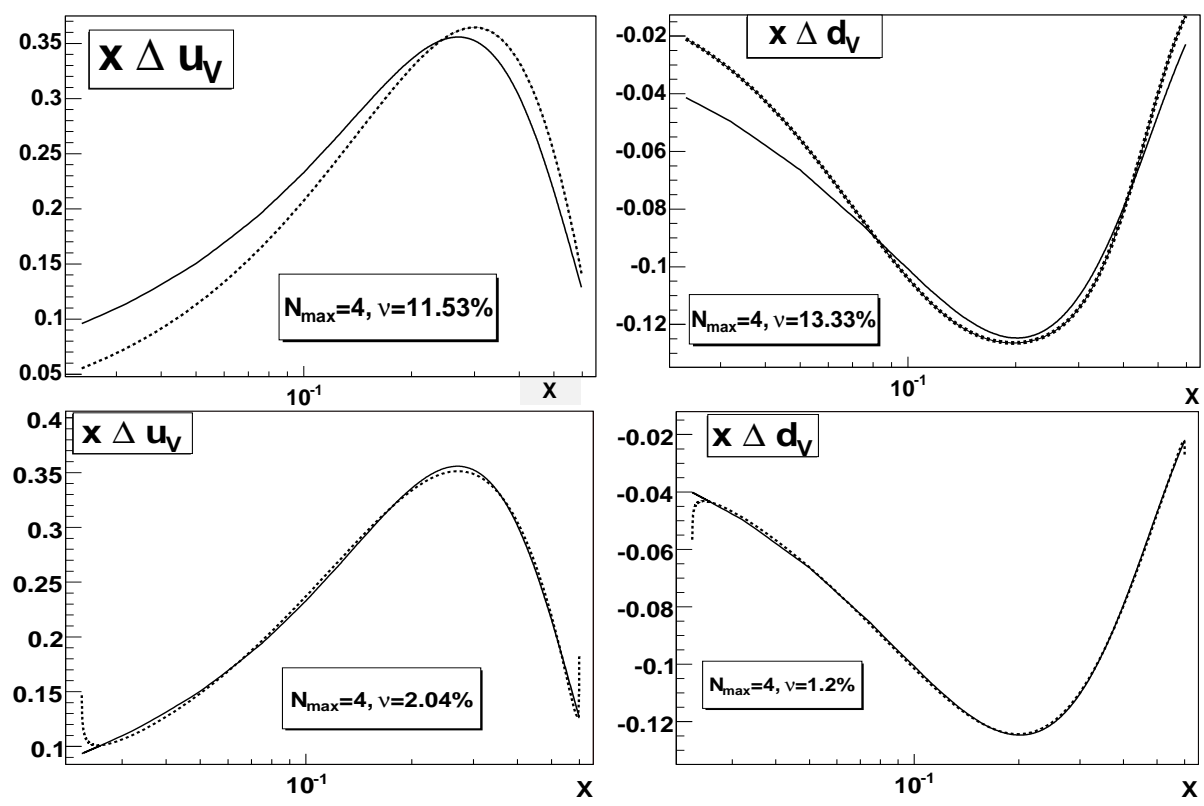
Figure 4: The results of $\Delta u_{V}$ and $\Delta d_{V}$ reconstruction for GRSV2000NLO parametrization for both symmetric (top) and broken sea (bottom) scenarios. Solid line corresponds to the reference curve (input parametrization). Dotted line is reconstructed with MJEM and criterium (10) inside the accessible for measurement region $([0.023,0.6]$ here $)$. Optimal values of parameters for symmetric sea scenario for $\Delta u_{V}$ are $\alpha_{\text {opt }}=-0.15555, \beta_{\text {opt }}=-0.097951$ and for $\Delta d_{V}$ are $\alpha_{\text {opt }}=-0.002750, \beta_{\text {opt }}=-0.07190$. Optimal values of parameters for broken sea scenario for $\Delta u_{V}$ are $\alpha_{\text {opt }}=-0.209346, \beta_{\text {opt }}=0.153417$ and for $\Delta d_{V}$ are $\alpha_{\text {opt }}=0.702699, \beta_{\text {opt }}=-0.293231$.
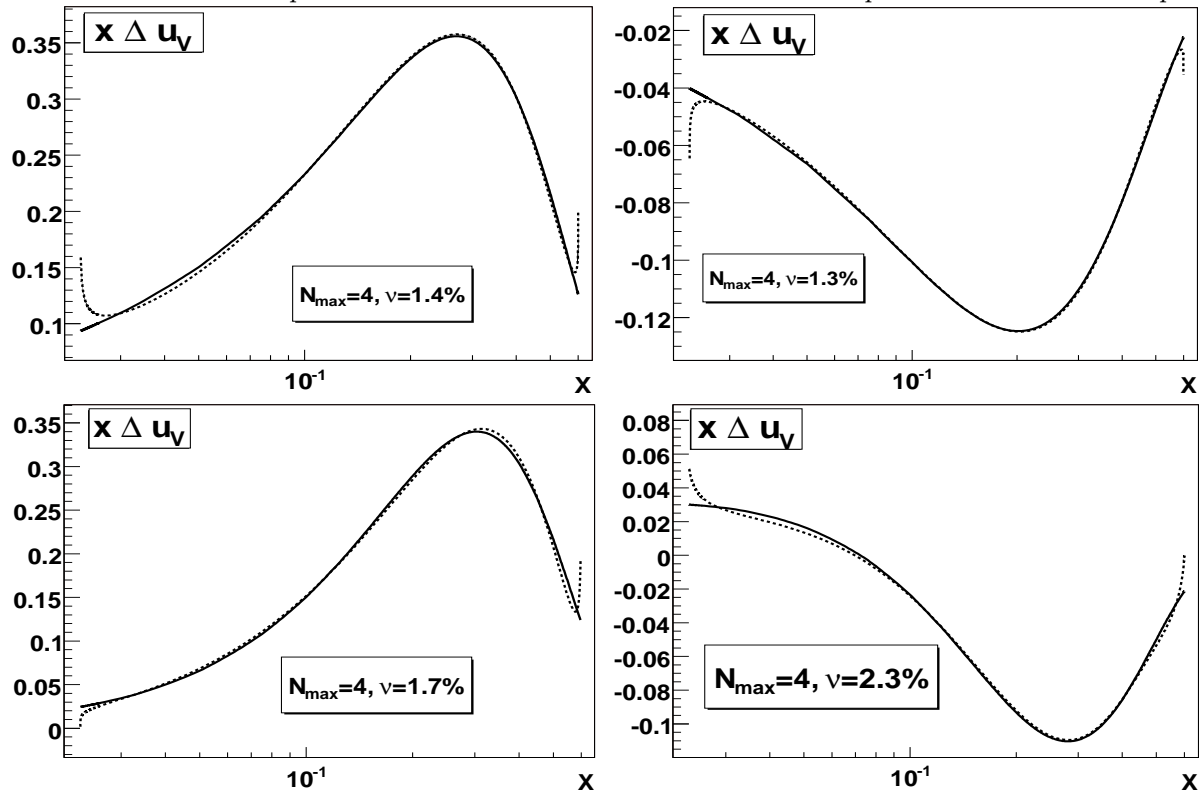
Figure 5: The results of $\Delta u_{V}$ and $\Delta d_{V}$ reconstruction in the region $\left[a_{\min }=10^{-4}, b_{\max }=1\right]$ for GRSV2000NLO parametrization for both symmetric (top) and broken sea (bottom) scenarios. Solid line corresponds to the reference curve (input parametrization). Dotted line corresponds to the curve reconstructed in the entire $\left[a_{\max }=10^{-4}, b_{\max }=1\right]$ region with requirement of minimal deviation from the curve (bold solid line ) reconstructed with MJEM and criterium (10) inside the accessible for measurement region $([0.023,0.6]$ here).
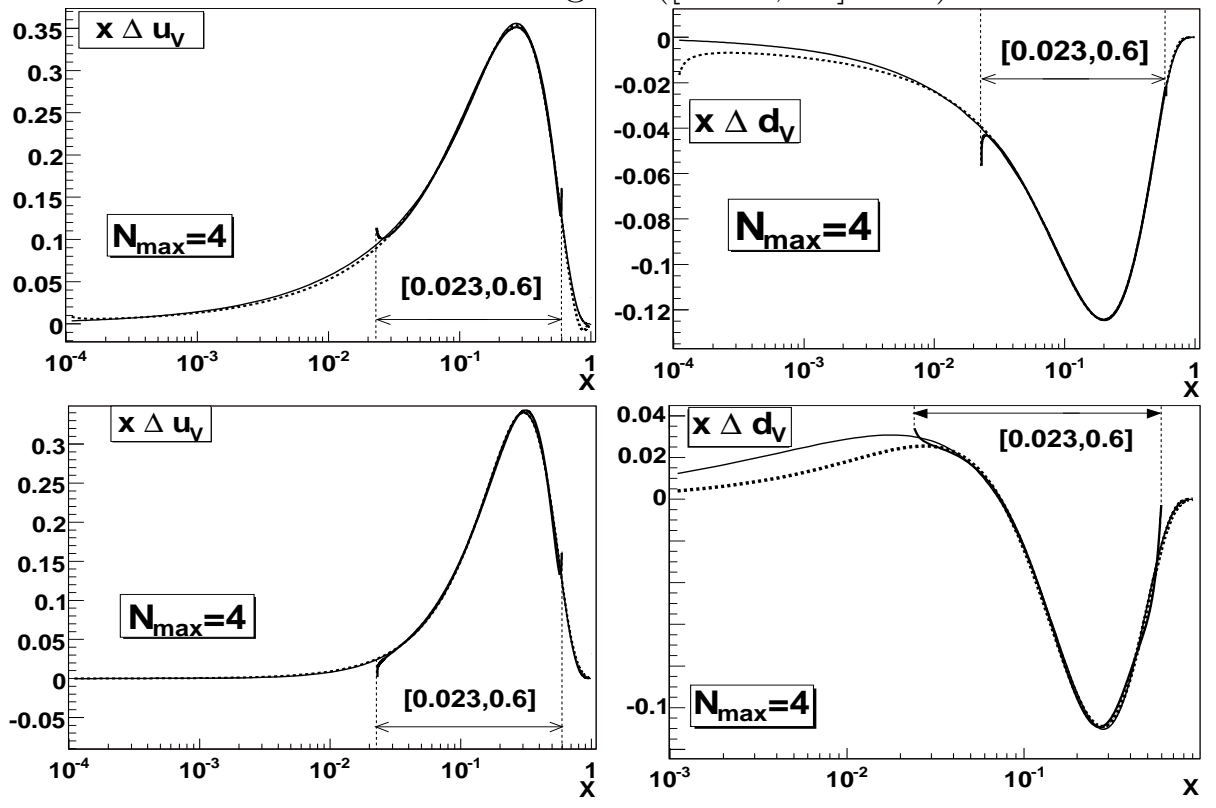